\title{
Contribuciones del posmodernismo y el post-estructuralismo al campo de la educación ambiental: Pesquisa y construcción
}

\author{
Edgar González-Gaudiano ${ }^{1}$ \\ Universidad Autónoma de Nuevo León, México
}

Resumo: El artículo analiza la vinculación existente entre el posmodernismo y el postestructuralismo en el campo de la educación ambiental y su extenso potencial en la investigación educativa. Se discute la diferencia entre posmodernismo y postestructuralismo en el marco de las críticas al proyecto modernista surgida de la Ilustración, en las que a partir de la función ideológica que desempeñan las metanarrativas en la configuración de una concepción del mundo, se revisan particularmente las nociones de verdad, de orden, de progreso y de sujeto como elementos significativos de una aproximación metodológica distinta. Finalmente, se analiza el concepto de pensamiento débil y su valor en la construcción de una propuesta paradigmática más acorde con las tesis postestructuralistas.

Palabras clave: post-estructuralismo; educación ambiental; investigación en educación ambiental.

Abastract: This paper analyses the links between postmodernism and post-structuralism in the environmental education field and their potential in education research. It also discusses the differences between postmodernism and post-structuralism criticisms towards the modern project provided by the Enlightenment. These new perspectives allow to examine the ideological role played by metanarratives in the construction of world views, particularly reviewing the notions of truth, order, progress and human subject as significant elements of a distinct methodological approach. Finally, the paper analyses the concept of weak thought and how much it is worth in the construction of a paradigmatic proposal more according to the poststructuralist ideas.

Keywords: post-structuralism; environmental education; environmental education research.

\footnotetext{
${ }^{1}$ E-mail: egonzalezgaudiano@gmail.com
} 


\section{Introducción}

Como hemos estado escuchando durante estos días en que se está celebrando el IV EPEA, el posmodernismo es un tópico nodal de discusión en las humanidades, así como en la teoría y la investigación social, incluyendo por supuesto la investigación educativa. Este tópico ha tenido una gran repercusión mundial, pero no tanto en América Latina donde se le ha visto con indiferencia e incluso irritación, quizá porque como dice Adriana Puiggrós, nuestros pueblos no acabaron nunca de entrar en la modernidad, ni mucho menos en cumplir sus promesas de desarrollo. Por ello me causó un poco de sorpresa la invitación para participar en este foro que estaría dedicado a discutir el posmodernismo en el marco de la investigación en educación ambiental.

\section{Las dos acepciones del posmodernismo}

Antes de comenzar esta exposición quiero aclarar dos aspectos que me han pedido desarrollar, que se condensan en la frase métodos de investigación posmodernista. Primero, porque para plantear lo concerniente a educación ambiental no hablaré precisamente desde el posmodernismo, sino del post-estructuralismo, pero me explico.

De acuerdo con Peters (1996), el término 'modernismo' tiene dos acepciones. La primera se refiere al fecundo movimiento en las artes iniciado desde finales del siglo XIX; la segunda acepción es más histórica y filosófica, remite a 'lo moderno', el periodo que siguió a la larga etapa medieval iniciado en el Renacimiento y alimentado por el inspirador momento de la Ilustración en los siglos XVII y XVIII. Existe relación entre las dos acepciones, ya que ambas - 'lo moderno' y el 'modernismo' - implican romper con lo caduco, lo clásico, lo tradicional, y ponen énfasis en lo nuevo o lo actual. Esta ruptura conlleva el sentido de que lo nuevo es mejor que lo viejo. Revisaremos esto más adelante.

De ahí que el 'posmodernismo' hereda estos dos significados relativos a los dos sentidos del término modernismo. Es decir, puede usarse estéticamente para referirse específicamente al desarrollo de las artes en reacción al modernismo (el avant-garde, el experimentalismo que 
siguió a la crisis de representación expresada a través del cubismo, dadaísmo y surrealismo; el proceso de creciente abstracción como el expresionismo abstracto o el minimalismo). Pero, puede usarse también en el sentido filosófico e histórico para describir críticamente el periodo posterior a la modernidad o a su ethos. En otras palabras, para representar la transformación de la modernidad o sea el giro radical en el sistema de valores y prácticas que subyacen en la modernidad.

\section{Posmodernismo o post-estructuralismo}

Lo anterior se vuelve más complejo cuando algunos pensadores post-estructuralistas comenzaron a involucrarse con el término posmodernismo. En específico Lyotard (1979), a partir de la publicación de 'La Condición Posmoderna', donde analiza el estatus del conocimiento en las sociedades más avanzadas provocando variadas rupturas con la era moderna y con sus formas de ver el mundo. Pero Lyotard define lo posmoderno simplemente como una 'incredulidad hacia las metanarrativas' y específicamente menciona a "la progresiva emancipación de la razón y la libertad, la progresiva o catastrófica emancipación del trabajo..., el enriquecimiento de todos a través del progreso de la tecnociencia capitalista e incluso... de la salvación de las creaturas mediante la conversión de las almas a la cristiana narrativa del amor por el martirio".

Las metanarrativas son grandes historias, relatos maestros, que las culturas cuentan de sí mismas — con base en sus prácticas y sus creencias-, con el fin de legitimarlas. Funcionan como una sencilla historia unificada que persigue justificar un conjunto de prácticas, una autoimagen cultural o institucional, frente a sí mismos y frente a los otros.

Los posmodernistas desconfían de las metanarrativas; sospechan de Hegel, Marx y de cualquier forma de filosofía universal. El advenimiento de la posmodernidad provocó que las grandes historias de la modernidad - la dialéctica del Espíritu, la emancipación del trabajador, la acumulación de riqueza, la sociedad sin clases - perdieran credibilidad, se erosionaran, se debilitaran, por lo que ya no tienen el consenso que tenían antes cuando fueron elevadas a la categoría de filosofías de la historia. 
A partir de la mitad del siglo pasado, las sociedades occidentales comenzaron a cambiar radicalmente. Para describir esos cambios los teóricos sociales han empleado varios términos: sociedad mediática, sociedad del espectáculo, sociedad de consumo, sociedad post-industrial. Una de esas descripciones ha sido sociedad posmoderna. Según Sarup (1989), Lyotard es un post-estructuralista que adoptó una postura posmodernista y cree que el posmodernismo es un nuevo tipo de sociedad, pero también otra forma para designar el post-estructuralismo. Yo no creo eso y estoy convencido de que a nivel académico es mucho más apropiado hablar de lo posmoderno en el sentido de una ruptura con lo moderno que, en palabras de Buenfil (1998), es un movimiento que ha contribuido a erosionar el carácter absoluto de los fundamentos modernos; pero para referirnos a un nuevo modo de pensar, un estilo de hacer filosofía, una nueva clase de crítica social, de militancia teórica, es mejor usar el término post-estructuralismo.

\section{Sobre el método}

La segunda cuestión que deseo aclarar es lo relativo a los métodos. Si bien el post-estructuralismo cuestiona severamente la racionalidad técnica e instrumental empleada en la investigación científica, en rigor no ofrece todavía métodos propios. Sin embargo, si examináramos algunos de los más importantes modos de análisis en el post-estructuralismo y me refiero a la arqueología, la genealogía, la deconstrucción y el post-estructuralismo feminista, podemos encontrar algunas pistas que pueden apoyarnos para desarrollar una nueva perspectiva de investigar y de hacer análisis y crítica educacional. Retomaré este asunto metodológico al final de mi exposición.

Así que una vez habiendo dicho sobre qué no voy a hablar, comencemos a exponer lo que sí he incluido. Por cierto, es típico en el enfoque post-estructuralista que antes de decir lo que sí es, se describe lo que no es. 


\section{La Ilustración y la investigación educativa}

El medio de la investigación educativa actual tiende a ser relativamente conservador, fue fundado y sigue estando fuertemente imbuido por el ethos positivista que heredó durante su desarrollo como un campo de estudio legitimado a partir de la Ilustración. El enfoque de la Ilustración - la creencia en un progreso racional, en el sujeto individual, en el orden monolítico y en la verdad universal- se encarnó en la concepción moderna de ciencia y obviamente en la investigación científica. Este enfoque considera, entre otros aspectos, que el mundo tiene un orden, el investigador sólo debe encontrarlo y esto está en relación directa con el tipo de conocimiento que se ha construido desde entonces.

El proyecto de la Ilustración surgió, como dije antes, en los siglos XVII y XVIII en Europa occidental y estuvo ligado a la emergencia de muchos otros grandes movimientos intelectuales e históricos, tales como la racionalidad, la ciencia experimental y la modernidad en sí misma.

Para los fines de esta presentación podemos definir qué es la Ilustración, tomando como punto de partida lo que señala Barnes (1996), para no embarcarnos en una exposición demasiado filosófica que no nos daría tiempo de desarrollar ni siquiera medianamente bien.

Primero, la Ilustración se caracterizó por la creencia en la inviolabilidad del sujeto humano. La noción 'sujeto' se refiere a algo distinto del término más familiar 'individuo'; proviene del Renacimiento y presupone que el hombre y la mujer son libres, son agentes intelectuales y que sus procesos de pensamiento no están coaccionados por circunstancias históricas y culturales. Es decir, se consideraba a los sujetos como autónomos, soberanos y autoconscientes. En general, se asume que hay una esencia irreductible, estable, desalienada en cada individuo humano. Esta creencia se expresó de variadas maneras en las distintas formas de humanismo. Pero en términos generales, lleva a la conclusión de que al haber esencias humanas, esto es, si cada sujeto individual es esencialmente lo mismo, lo que sirve para uno sirve para todos y de ese modo los juicios acerca de lo bueno, lo verdadero y lo hermoso son generalizables, se universalizan, se convierten en verdades universales, también reconocidos simplemente como universales. Por ejemplo, ¿no han notado que en los 
patrones estéticos aplicados en los concursos de belleza predominan los rasgos occidentales?

Tal perspectiva de la Razón es clara en el trabajo filosófico de Descartes. Consideremos la idea manifiesta en el cógito cartesiano: "Pienso, luego existo". El 'Yo' de Descartes se asume plenamente consciente y por lo tanto autocognoscente. Es no sólo autónomo sino coherente; no se concibe la posibilidad de que haya otro territorio psíquico, que pudiera contradecir a la conciencia.

Desde la perspectiva estructuralista, por ejemplo, Levi-Strauss (1908-presente), quien llamó al sujeto humano -el centro del ser- 'el mocoso mimado de la filosofía', sostenía que el fin último de las ciencias humanas no es constituir al hombre sino disolverlo.

Los post-estructuralistas como Foucault (1926-1984) quisieron deconstruir estas concepciones; el término 'sujeto' ayudó a concebir la identidad como una construcción, como un producto de actividades significantes, culturalmente específicas y generalmente inconscientes. La categoría de sujeto en la perspectiva post-estructuralista introduce una noción del yo (self) que 'descentra' la conciencia y disuelve el sujeto. En un cierto sentido puede decirse que Derrida (1930-2004) y Foucault (19261984) no construyeron una 'teoría' del sujeto. La excepción es Lacan (1901-1981), quien sí se compromete con el análisis del sujeto por su formación filosófica Hegeliana y su compromiso con el psicoanálisis; de ahí su aportación de lo que se reconoce como el triángulo lacaniano integrado por lo real, lo simbólico y lo imaginario, así como sus trabajos sobre el sujeto escindido, dividido, el sujeto de la renuncia.

Segundo, la Ilustración promovió la creencia en la noción de progreso, que está vinculada a la aplicación de la racionalidad y la razón. Esta idea sostiene que el mundo ha caminado siempre hacia delante. Así, la Teoría de Newton es sólo un caso especial de la de Einstein. Más simplemente, el siglo XXI es mejor que el XX y ese fue mejor que el XIX, porque las mismas cosas son hechas con más eficiencia, con más rigor lógico, esto es más racionalmente que en el pasado.

El post-estructuralismo hace una crítica de este historicismo lineal, rechazando el fundamento que afirma que existe un modelo global de la historia. Es decir, cuestiona acremente las tesis que sostienen que la 
historia se mueve en una dirección tal que el momento presente es superior al pasado (lo nuevo mejor que lo viejo). Por ejemplo, Foucault escribe sobre la historia sin esta noción de progreso y Derrida dice que no hay un punto final en la historia. Recuerden cuando Francis Fukuyama, frente a la caída de la Unión Soviética, declaró el fin de la historia.

Desde esta perspectiva lineal y ascendente de progreso, la educación para el desarrollo sustentable es mejor que la educación ambiental simplemente porque es una formulación temporalmente más reciente, por lo que supera los sesgos y limitaciones de la educación ambiental.

Tercero, también se fomentó la creencia de que el mundo tenía un orden y que la tarea de la ciencia era encontrarlo. Ya he mencionado antes un poco esto, es decir, detrás de la aparentemente efímera y evanescente experiencia humana y en todo lo que constituye el mundo real, hay un orden oculto. La clave otra vez es la racionalidad: así se desarrollaron los métodos racionales en cuyo pináculo estaban la lógica y las matemáticas, el Prof. Gallo habló también de esto durante su presentación hace un par de días. La consecuencia de estos métodos fue la totalización. Si bien podíamos ver al mundo como un conjunto de cosas desconectadas, la realidad subyacente era que había coherencia y totalidad, sostenida por el poder superior de la racionalidad. Hablaremos de este asunto un poco más adelante porque es un asunto del cual fácilmente podemos inferir que está en relación directa con el conocimiento y la causalidad.

Finalmente, la creencia en la verdad universal, es decir que hay una verdad válida para todo tiempo y lugar. Para adquirir esa verdad, es necesario hacer referencia a los fundamentos o esencias. Esas esencias son las piedras de toque que nos pueden permitir alcanzarla. Las esencias para el cartesianismo, el cogito, ergo sum; para el positivismo lógico, las piezas del mundo reflejadas en el lenguaje y para el marxismo, la posición de clase y en particular la de los trabajadores era esencial para establecer la verdad histórica de la muerte del capitalismo.

Aunque diferentes en sustancia cada una de estas epistemologías (narrativas maestras) adoptó la misma estrategia, encontrar una premisa irrebatible y usarla como base de una construcción racional de un sistema 
de conocimiento infalible. Es lo que mencionamos al principio de la exposición sobre las metanarrativas o narrativas maestras.

\section{Estructuralismo y post-estructuralismo}

Durante los últimos cincuenta años, el estructuralismo y el postestructuralismo revolucionaron los paradigmas prevalecientes relacionados con la comprensión de lo humano surgidos de la Ilustración. Levi-Strauss, Lacan, Derrida, Foucault, Deleuze, Lyotard, Baudrillard, Vattimo y Laclau, entre otros, produjeron $-\mathrm{y}$ algunos de ellos siguen produciendoimpresionantes contribuciones. El pensamiento estructuralista y postestructuralista son diferentes entre sí, pero ambos son enfoques críticos, aunque el post-estructuralismo está comprometido con la crítica de las instituciones dominantes y los modos de habla, pensamiento y escritura.

Por ejemplo, el post-estructuralismo ha impulsado una crítica del significado. Tema fundamental en la investigación. Mientras en Inglaterra se había dado una fuerte influencia por las teorías del lenguaje durante los primeros años del siglo veinte (Wittgenstein, Ayer, etc.) en Francia se retrasó la entrada del giro lingüístico en la filosofía francesa. Recordemos que Saussure enfatizaba la distinción entre significante y significado. El sonido hecho al pronunciar la palabra 'manzana' es el significante, y el concepto de manzana es el significado. La relación estructural entre el significante y el significado constituye un signo lingǘstico, y esto es importante entenderlo porque el lenguaje está hecho de signos lingüísticos. El signo lingüístico es arbitrario; esto significa que se establece para algo por convención y uso común, no por necesidad. Saussure también señaló que cada significante adquiere su valor semántico sólo en virtud de su posición diferencial dentro de la estructura del lenguaje. En esta concepción del signo hay un balance precario entre significante y significado. En el sujeto portador, ampliamente hablando, el significado es degradado y el significante se vuelve dominante. Esto implica que no hay una correspondencia uno a uno entre proposición y realidad.

Desde el post-estructuralismo, Lacan (1901-1981), por ejemplo, escribe sobre 'el incesante deslizamiento del significado bajo el significante’. Derrida va más lejos: el cree en un sistema de significantes 
flotantes puro y simple, que no tiene una relación determinable por ningún referente extralingüístico.

Pero ¿Qué es un significante flotante? Lo explicaré muy brevemente aunque utilizamos los significantes flotantes todo el tiempo en nuestra habla cotidiana. Pensemos en la palabra democracia, o en algo más próximo a este colectivo, la palabra conservación.

Conservación es un significante cuyo significado ha estado asociado a la Naturaleza (otro significante con el que va constituyendo una cadena de significación). Si debemos conservar sólo los espacios naturales, la vida silvestre, eso implica que no hay conservación en aquello que está modificado. Este significado se va degradando de manera que ya no tengo que hablar de conservación de la naturaleza, para entender que conservación implica eso con todos sus sesgos implícitos. Este desplazamiento se encuentra influyendo en las políticas ambientales, las concepciones de educación ambiental, etc. El significante flota entre un discurso a otro y al flotar se va vaciando de contenido convirtiéndose en un significante vacío. El ser vacío no significa que no tenga significado sino que incorpora de manera precaria y provisional el significado que se establece en relación con los otros elementos del discurso. Es un significado particular, propio, que no llena completamente al significante, pero lo fija temporalmente y así se convierte en un punto nodal que genera tensión dentro de un campo, pero fija, detiene temporalmente, el flujo de significaciones en ese campo. No tenemos tiempo para explicar más esto, pero resulta importante, por ejemplo, para entender las propuestas metodológicas de análisis de discurso (conceptual, educativo, político, etc.).

Por otro lado, si bien el estructuralismo ve a la verdad 'atrás' o 'dentro' de un texto, el post-estructuralismo apunta hacia la interacción del lector y al texto como productividad. En otras palabras, la lectura pierde su estatus de consumo pasivo de un producto y se convierte en ejecución (performance).

Resumiendo, el post-estructuralismo critica la concepción clásica cartesiana de sujeto unitario - el sujeto/autor como productor de conciencia, autoridad para el significado y la verdad; el sujeto no tiene una conciencia unificada sino que es estructurada por el lenguaje. Así, el post- 
estructuralismo formula una crítica a la metafísica de la presencia, al concepto de causalidad, de identidad, de sujeto, de verdad.

\section{La construcción del conocimiento}

En 'La Condición Posmoderna', Lyotard (1979) aduce que durante las últimas décadas las ciencias dominantes y las tecnologías se han involucrado con el lenguaje: teorías de lingüística, problemas de comunicación y cibernética, computadoras y sus lenguajes, problemas de traducción, almacenamiento de información, y bancos de datos.

La transformación tecnológica ha tenido un considerable impacto sobre el conocimiento. La miniaturización y comercialización de maquinas está cambiando la forma en la que el aprendizaje es adquirido, clasificado, puesto a disposición y aprovechado. Consideremos los avances en la investigación genómica y nanotecnológica, por ejemplo, con todas sus implicaciones ambientales, bioéticas, etc. Lyotard cree que la naturaleza del conocimiento no puede sobrevivir sin cambios dentro de un contexto de transformación general, por lo que el status de conocimiento se altera a medida que las sociedades van entrando en la era posmoderna. Predijo que aquello que en el cuerpo constituido de conocimiento no sea traducible en cantidades de información será abandonado y que la nueva investigación será dictada por la posibilidad de que su resultado sea traducible a un lenguaje computacional. Con todo esto, el conocimiento está dejando de ser un fin en sí mismo, sino que se produce para ser vendido.

Es ampliamente aceptado que el conocimiento computarizado ha modificado la noción fuerza de producción en las últimas décadas. La fuerza de trabajo se ha modificado en los países, ha disminuido la cantidad de gente en las fábricas y en el campo, y se ha incrementado en trabajadores profesionales, técnicos y de cuello blanco. El conocimiento y la sociedad del conocimiento será la mayor competencia en el mundo por el poder y el control de la información, justo como se combatió antes por nuevos territorios. En la era posmoderna la ciencia se fortalecerá como preeminencia en el arsenal de capacidades productivas de los estados-nación y la brecha entre desarrollados y en desarrollo se hará mucho más grande. 
Vattimo, el filósofo italiano del que habló también el Prof. Gallo, concuerda con Lyotard, puesto que sostiene que la modernidad ya tuvo su momento. Así, el post-modernismo debe verse como un momento posterior a la modernidad y esto implica, sobre todo, como un momento posterior a las concepciones construidas en la modernidad como es el caso, ya mencionado, de la concepción de la historia como curso unitario, lineal y progresivo en el cual lo 'nuevo' es sinónimo de 'mejor'. Esto, por ejemplo, debe remitirnos al tema de las innovaciones educativas que se nos presentan como propuestas presuntamente superiores en términos de calidad, eficiencia y rigor.

Por eso es que Vattimo (1996) anuncia el fin de 'La Sociedad Transparente, en la que los mass media que ahora circundan y envuelven toda nuestra experiencia cotidiana, han contribuido a develar las bases de esa historia centrada en el ideal europeo de humanidad, una historia sostenida en una concepción de ciencia movida más por lo político y sus intereses extracientíficos, que por los fundamentos en los que se dice sostener; una historia que ha ordenado el mundo en función de coordenadas europeas. Recuerden por ejemplo, que el meridiano cero, se encuentra en Greenwich, en las afueras de Londres. De igual modo, la concepción de que el Norte está arriba y el Sur abajo es una concepción ideológica-política que responde a los intereses de la órbita Noroccidental.

$\mathrm{Y}$ así esas grandes historias, cuentos, mitos, que ha propagado la edad moderna, si bien no están del todo superados, están en ese proceso, encontrándonos en lo que Vattimo llama el tránsito de un pensamiento fuerte a un pensamiento débil.

En la presentación del Prof. Gallo se comentó lo de las ciencias menores, que suscitaron varias preguntas del público. Esto se encuentra en relación con el pensamiento débil. Expliquemos. Vattimo denomina pensamiento fuerte o duro a aquel que habla en nombre de la verdad, de la unidad y de la totalidad (o sea, aquel pensamiento, como el de la ciencia moderna, que establece las bases definitivas y universales del conocimiento y la intervención). Una metanarrativa, según el Posmodernismo.

Consecuentemente, Vattimo concibe el pensamiento débil como aquel pensamiento que rechaza esos fundamentos universales, definitivos, es decir, duros (en términos de fuerza y duración), así como también los 
argumentos normativos totalizantes, esencialistas, omnicomprensivos, que han sido legitimados en el devenir de la racionalidad surgida en la modernidad.

Por pensamiento débil no debe entenderse un pensamiento peyorativamente de menor grado, sino un pensamiento de distinta clase que apela a una condición diferente, en la que ya no se busquen esencias, ni fundamentos estables; un pensamiento que sospeche de las pretensiones de universalidad, de las respuestas generalizables a todo momento y todo lugar. Debe verse, dice Vattimo (1996), como una forma de nihilismo, "una especie de destino del que no podemos liberarnos sin privarnos de aspectos fundamentales de nuestra espiritualidad". Así, a diferencia de las críticas hechas al nihilismo por el pensamiento fuerte, este debe reconocerse como una posibilidad, puesto que aceptar que no tenemos más que vivir en la incertidumbre, en la ausencia de fundamentos, de pisos firmes y estables, sin garantías y, es por eso, que Vattimo hereda de Nietzsche la idea de "la muerte de Dios", que tanto conmovió a la filosofía occidental, que se refiere precisamente a la carencia de esas verdades, esos fundamentos, esos valores absolutos.

De algún modo, pienso que ya están percatándose de las enormes implicaciones que esta ruptura epistemológica tiene en el campo de la educación en general y de la educación ambiental en particular. Esta concepción post-estructuralista nos ofrece la oportunidad de trabajar el conocimiento como proceso y no como producto, como suele emplearse en las aulas; pero proceso no en el sentido de camino necesariamente ascendente, lineal y progresivo, sino en el sentido de ser construido socialmente en perspectivas no jerárquicas, ni universalizantes, ni definitivas. Y esto implica grandes posibilidades para un campo como el de la educación ambiental que desea establecer diálogos de saberes y entre disciplinas, de construir aproximaciones que nos permitan formular otras explicaciones y comprensiones sobre nuestro papel en y con el mundo sin pretensiones hegemonizantes. Este pensamiento débil puede permitirnos deconstruir la centralidad que prevalece en el conocimiento sobre la educación y sobre el ambiente e intentar construir otra configuración de fuerzas, nuevos puntos nodales que nos ayuden a cambiar los sesgos de la educación ambiental.

Pero hay que tomar conciencia de que esto no va ser fácil, porque nos resistimos a la idea de que no hay bases firmes para el pensamiento y 
la acción, sino que se trata de 'fundamentos' fluidos, faltos de certezas y eso suele producirnos ansiedad y desasosiego.

Veamos, por ejemplo, qué implicaciones puede tener todo esto para el tema metodológico que provocó que yo fuera invitado a hablar ante Uds. El post-estructuralismo puede permitirnos explorar desde la perspectiva deconstructiva la manera como se han sedimentado en la educación ambiental ciertas concepciones teóricas y prácticas ligadas a la enseñanza de las ciencias, a ver al ambiente como naturaleza y a la conservación como una estrategia de política ecológica que no otorga valor a las poblaciones humanas. Un asunto de primer orden para la educación ambiental ¿no creen? Porque si no somos capaces de identificar los componentes principales que se mueven en el discurso de la conservación en nuestros contextos de trabajo, podemos estar contribuyendo precisamente a posicionar los intereses que supuestamente estamos queriendo combatir. $\mathrm{Y}$ así nuestro trabajo se convierte más en parte del problema que de la solución.

El análisis deconstructivo produce fisuras que descentran y hacen más visibles esos componentes discursivos, identificando las lógicas, la dirección y la intención que tienen los campos discursivos, y sus entramados de significación que le dan sentido. Y aquí no estoy entendiendo la noción de discurso como una proclama grandilocuente, sino como una configuración de significados en permanente movimiento con elementos verbales y no verbales, que está sujeta a la historicidad y atravesada por la ambigüedad de sentido. Lamentablemente no pude abordar esto por razones de tiempo, pero lo podemos agendar para mi próxima visita.

A través de las estrategias genealógicas desarrolladas por Foucault podemos, por ejemplo, analizar diacrónicamente las implicaciones institucionales y de poder que determinaron el surgimiento de la noción educación para el desarrollo sustentable y la declaratoria de la década misma. Es decir, analizar a qué responde una educación que se encuentra ligada a una noción ambigua, confusa y con pretensiones universalizantes y que, de acuerdo con Isabel Carvalho, Gabriela Scotto y Leandro Guimarães, pone de manifiesto que se trata de un campo de disputa sobre diferentes concepciones de sociedad. 
También podemos revisar ¿Cómo funcionan las instituciones escolares a nivel curricular y en sus procesos de gestión en relación con los temas ambientales? Ello para develar las contradicciones existentes, pero también las condiciones de posibilidad de intervenir con mejores resultados en esos procesos.

En fin, creo que estoy muy excedido en el tiempo que generosamente me han concedido y sólo espero poder despertar en Uds. la inquietud por explorar y formarse en este pensamiento que ha dislocado cualitativamente nuestros fundamentos $\mathrm{y}$ ha contribuido a disolver las esencias en las que tanto creímos.

\section{Referencias}

BARNES, Trevor J. Logics of dislocation. Models, metaphors, and meanings of economic space. New York, The Guilford Press, 1996.

BUENFIL, Rosa Nidia (Coord.) Debates políticos contemporáneos. En los márgenes de la modernidad. México, Plaza y Valdés, 1998.

DE ALBA, Alicia (Comp.). Posmodernidad y educación. México: CESU-UNAM-Porrúa, 1995. (Colección Problemas Educativos de México).

GRANJA CASTRO, Josefina (Comp.). Miradas a lo educativo. Exploraciones en los límites. México: Plaza y Valdés, 2003.

JIMÉNEZ GARCÍA, Marco Antonio (Coord.). Los usos de la teoría en la investigación. México: Plaza y Valdés, 2006.

LYOTARD, Jean-François. La Condición Posmoderna. Madrid, Cátedra [1979]. 1998.

PETERS, Michael (1996) Poststructuralism, politics and education. Wesport, Bergin \& Garvey, 1996.

PETERS, Michael A.; BURBULES, Nicholas C. Poststructuralism and educational research. Lanham, Maryland: Rowman \& Littlefield Pub., 2004. (Philosophy, Theory, and Educational Series).

SARUP, Madan. An introductory guide to poststructuralism and postmodernism. Athens, Georgia: The University of Georgia Press, 1989.

VATTIMO, Gianni. La Sociedad Transparente. Barcelona, Paidós-Ibérica.-ICE-UAB, 1996.

Artigo recebido em 17/10/2007 - Aprovado em 30/05/2008 